\title{
High-frequency neuronavigated rTMS effect on clinical symptoms and cognitive dysfunction: a pilot double-blind, randomized controlled study in Veterans with schizophrenia
}

Heng Yong Guan', Jian Min Zhao', Ke Qiang Wang1, Xiu Ru Su', Yan Fen Pan', Jin Ming Guo', Long Jiang ', Yu Hong Wang ${ }^{1}$, Hong Yu Liu', Shi Guang Sun', Hao Ran Wu', Yan Ping Ren², Han Song Geng ${ }^{1}$, Xiao Wen Liu', Hui Jing Yu' ${ }^{1}$, Bao Chun Wei ${ }^{1}$, Xi Po Li ${ }^{1}$, Hanjing Emily Wu ${ }^{3}$, Shu Ping Tan ${ }^{4}$, Mei Hong Xiu ${ }^{4}$ and Xiang Yang Zhang ${ }^{5,6}$

\begin{abstract}
Cognitive impairment is a central aspect of schizophrenia (SCZ) that occurs at the onset of the disease and is related to poor social function and outcome in patients with SCZ. Recent literatures have revealed repetitive transcranial magnetic stimulation (rTMS) to be one of the efficient medical interventions for cognitive impairments. However, no study has been conducted to investigate the treatment effectiveness of $20 \mathrm{~Hz}$ rTMS with neuronavigation system administered to the left dorsolateral prefrontal cortex (DLPFC) in patients with schizophrenia. In this randomized, double-blind and sham-controlled study, 56 patients were enrolled in $20 \mathrm{~Hz}$ rTMS $(n=28)$ or sham stimulation ( $n=28$ ) over left DLPFC for 8 weeks. The Repeatable Battery for the Assessment of Neuropsychological Status (RBANS) was performed to measure the cognitive function at baseline and after 8 weeks of rTMS treatment. The positive and negative syndrome scales (PANSS) was performed to assess the clinical symptoms at baseline, after 2-week treatment, 4-week treatment, 6-week treatment, and 8-week treatment. Totally, 15 subjects (seven in active group and eight in sham group) dropped out during the trial and the main findings were from completed 41 patients. At 2 weeks, 4 weeks, and 6 weeks, there were no significant differences in PANSS total score and subscores between the sham and treatment groups. At 8 weeks, the $20 \mathrm{~Hz}$ rTMS significantly increased the immediate memory score compared with the sham. Furthermore, the improvement in the immediate memory score was correlated with the decrease in the excitement factor score of the patients with SCZ. Our results suggest that $20 \mathrm{~Hz}$ rTMS appears to be an effective treatment for improving the cognitive performance and reducing the clinical symptoms of patients with SCZ.
\end{abstract}

\section{Introduction}

Schizophrenia (SCZ) is a chronic mental illness that affects about $1 \%$ of the whole population. Many antipsychotic medications have a good effect on the vast

\footnotetext{
Correspondence: Mei Hong Xiu (xiumeihong97@163.com) or

Xiang Yang Zhang (zhangxy@psych.ac.cn)

${ }^{1}$ Hebei Province Rong-Jun Hospital, Baoding, China

${ }^{2}$ The National Clinical Research Center for Mental Disorders \& Beijing Key Laboratory of Mental Disorders, Beijing Anding Hospital, Capital Medical University, Beijing, China

Full list of author information is available at the end of the article.
}

majority of positive symptoms of patients in any phase of illness, but limited effect on negative symptoms or cognitive dysfunction ${ }^{1}$. Negative symptoms in SCZ are characterized by low motivation, lack of speech, and little interest in social behavior. Particularly, patients with SCZ display a general impairment in cognitive function including working memory, executive function, selective attention, immediate memory, and delayed memory ${ }^{2-4}$. Both cognitive impairment and negative symptoms strongly and negatively impact daily functioning and 
determine social functioning throughout the lives of the patients with $\mathrm{SCZ}^{5}$. However, the current antipsychotic treatments have almost little or no effect on these symptoms, in particular cognitive impairment ${ }^{6}$.

Repetitive transcranial magnetic stimulation (rTMS) is recognized as an augmentation therapy for some symptoms of SCZ, who failed multiple pharmacologic interventions. rTMS has been shown to be effective in numerous severe neuropsychiatric diseases such as bipolar disorder, depression, and anxiety ${ }^{7}$. In SCZ, several double-blind, randomized control trials have been employed to investigate the effect of high-frequency rTMS treatment to stimulate the excitability of left dorsolateral prefrontal cortex (DLPFC) on negative symptoms of $\mathrm{SCZ}^{8-10}$. For example, a recent meta-analysis including 827 participants reported that the rTMS treatment target on the frontal cortex had a mean weighted effect size (ES) of $0.64^{11}$. In addition, the study also suggested that rTMS is effective in treating negative psychopathological symptoms in $\mathrm{SCZ}^{11}$. However, an earlier meta-analysis revealed no statistically significant difference in reduction of negative symptom scores between active rTMS group and sham group ${ }^{12}$. Collectively, all these results showed that further rTMS studies for systematically assessing psychiatric symptoms in certain subtypes of SCZ are warranted due to significant differences across numerous participants recruited in the different studies.

Conversely, only a few of studies reported the effects of rTMS treatment on cognitive dysfunction in $\mathrm{SCZ}^{13-15}$. For example, an open-label study showed that rTMS targeting to the left DLPFC $(10 \mathrm{~Hz})$ and left temporoparietal cortex $(1 \mathrm{~Hz})$ significantly improved auditory verbal memory ${ }^{16}$. Wolwer et al. indicated that $10 \mathrm{~Hz}$ rTMS stimulation to the left DLPFC significantly improved facial emotion recognition in $\mathrm{SCZ}^{17}$. In particular, rTMS treatment bilaterally sequentially to left and right DLPFC was shown to significantly improve the accuracy of 3-back test compared with sham ${ }^{15}$. However, the recent double-blind, sham-controlled trials of rTMS in patients with SCZ showed that $10 \mathrm{~Hz}$ rTMS targeting to the left DLPFC for 3 weeks was not superior to sham rTMS in the improvement of numerous cognitive domains ${ }^{18}$. Moreover, a most recent meta-analysis demonstrated that prefrontal rTMS exerted beneficial effects on attention and executive deficits in certain depressive patients but not in the patients with $\mathrm{SCZ}^{19}$. It should be noted that the treatment protocols regarding rTMS efficiency are not consistent. This may explain the dramatically significant difference between studies reported in prior meta-analyses. For example, technical differences in rTMS treatment protocols including the localization of treatment (left versus right versus bilateral), duration of treatment, stimulation frequency, accuracy of targeting, and patient characteristics may lead to different treatment effect of rTMS $^{11,20}$. Previous studies have focused primarily on stimulation frequencies of $10 \mathrm{~Hz}$ rTMS for 4 weeks, but found to be less effective in SCZ, especially for cognitive dysfunction. It is still unclear whether more repetitions or increased number of total pulses yield better results. Longer course of treatment and higher frequency of stimulation indicate more doses and more pulses of rTMS on DLPFC of the patients.

Due to the anatomic variation between patients, rTMS efficacy may be improved through a precise target ${ }^{21}$. For instance, most studies have targeted to the left DLPFC due to its central role in working memory and in executive function ${ }^{22}$. However, DLPFC is a comparatively large cortical area, which is neither accurate nor easy to target at this anatomical location. To date, most rTMS studies target based on the approximated "5-7- $\mathrm{cm}$ methods", which have proposed to target 5-7-cm anterior to the primary motor cortex ${ }^{23-25}$. However, literatures have revealed that the vast majority of treatment targets may not be accurate in the DLPFC ${ }^{26,27}$. Thus, further studies are needed to optimize rTMS targeting techniques to improve treatment efficacy. Neuronavigation system is an indispensable instrument for accurate planning, targeting, and monitoring in brain stimulation studies. Using rTMS, the system co-registers the patient's head to a standardized brain ${ }^{28}$. Under the guidance of the navigation system, the technicians can exactly observe the relative location of the magnetic coil of rTMS to the individual's brain. The entire stimulation process is completely visualized, and the detailed parameters of each stimulus can be saved as images. Therefore, the neuronavigation system allows for precise, optimal, and reproducible targeting and stimulation of the DLPFC sites.

Several recent literatures have shown some efficacious results by combining the high-frequency rTMS with neuronavigated target on auditory verbal hallucinations in SCZ, relative to sham stimulation ${ }^{21}$. This study was the first one to assess the treatment efficiency of $20 \mathrm{~Hz}$ rTMS for the clinical symptoms and cognitive dysfunction in patients with SCZ. We proposed that: (1) the combination of neuronavigation, high-frequency (e.g., $20 \mathrm{~Hz}$ ) stimulation and longer treatment duration ( 8 weeks) may be an optimal strategy for improving cognitive function and reducing negative symptoms in patients with $\mathrm{SCZ}$; (2) the rTMS-induced cognitive improvement may be associated with changes in certain symptoms that share a similar, yet separable etiology to the cognitive impairment.

\section{Methods \\ Participants}

All participants were recruited in HeBei Province RongJun Hospital in Northern China. The clinical trial protocol was approved by the Institutional Review Board of HeBei 
Province Rong-Jun Hospital. After receiving the full explanation on the purpose of the current study, every participant signed written informed consent form prior to recruitment. Fifty-six inpatients of ages 20-60 were recruited and diagnosed with SCZ by the Structured Clinical Interview for DSM-IV, without any patients with schizoaffective disorder. The patients also met the following inclusion criteria: (1) male; (2) right-handed; (3) Han Chinese; and (4) positive score on positive and negative syndrome scale (PANSS) $<24$ and PANSS negative symptom score $\geq 20$; and (5) $\geqq 5$-year duration of illness.

At baseline, we recorded a complete medical history of all patients. Recruited participants had no recent life stressors or clinically significant emotional abnormalities for at least 1 month prior to participation in the current study, which were obtained through the questions we asked them verbally. Subjects with physical diseases or cerebral pathologies including multiple sclerosis seizure, dementia, epilepsy, aneurysm, Huntington's disease, brain tumor, stroke, Parkinson's disease, severe headache for unknown reasons, and cardiovascular diseases were excluded. Also, those who received electroconvulsive therapy or rTMS within the past 6 months were excluded. Past history of autoimmune diseases, allergies, hypertension, lung disease, diabetes, cerebrovascular disease, family history of epilepsy, pregnancy or breast feeding, education level $<5$ years by subject report, receiving or planning to start the psychotherapy during the rTMS treatment, or those who had received psychotherapy in the last 6 months before the current study were also excluded.

\section{Procedure}

This study was a double-blinded and randomized controlled pilot trial. Of the 56 participants, 28 were in the active rTMS group $(20 \mathrm{~Hz})$ and 28 in the sham stimulation group. Briefly, subjects were assigned to receive 40 treatments of either $20 \mathrm{~Hz}$ or sham rTMS over the duration of 8 weeks.

After the recruitment, all participants were randomly and separately assigned to real $(20 \mathrm{~Hz})$ or sham rTMS condition. An independent, third party assorted participants into either the real or sham groups through computer generated randomization numbers that were compiled through simple randomization. The clinical staff and patients were blind to the assignment, except for one clinical technician, who provided the real rTMS or sham treatment according to the randomization numbers. Clinical assessment was performed at baseline and weeks $2,4,6$, and 8. Cognitive performance was assessed at baseline and week 8 . Raters who were blind to treatment status assessed clinical symptoms and cognitive function in the study.

\section{Neuronavigated rTMS treatment}

For the exact stimulation locations of the left DLPFC, neuronavigation system (Polaris Vicra position sensor, BrainSight, Magstim, UK) was used for accurate planning, targeting, and monitoring. Brain images for each patient were collected with a $0.3 \mathrm{~T}$ magnetic resonance imaging (MRI) (BrivoMR235). The image data were imported to the neuronavigation system and then the treatment positions were accurately located accordingly. All processes were performed by a clinical technician.

In active group, $20 \mathrm{~Hz}$ stimulus on left DLPFC occurred at a power of $110 \%$ of motor threshold for 3-s intervals with 27-s inter-train interval. Forty sessions of treatment were administered five times a week (Monday to Friday) for 8 weeks using a MagStim Rapid stimulator (total stimuli $=64,000$ ) (MagStim Company Ltd). In the sham group, all treatment procedures were the same as those in the $20 \mathrm{~Hz}$ group except for a false coil (P/N: 3950-00, Magstim Co.), which is different from the MagVenture CoolB65 Active/Placebo coil (P/N: 9016E0501; MagVenture $\mathrm{A} / \mathrm{S}$ ) in the intensity and distribution of the E-field in the subject's head ${ }^{29}$. The false coil was placed in the same position as the active treatment. The sham treatment produced the same vibration as the true stimulus but no magnetic field and thus no therapeutic effect. Both real and sham administrations are identical in appearance and in sound.

All patients were on a stable dose of antipsychotic medication for at least 6 months before the study enrollment, which means that the doses of antipsychotics remained fixed for at least 6 months. Moreover, antipsychotics and all other medications remained fixed throughout the double-blind period. The medications that patients had been taking were clozapine (16 in rTMS versus 20 in sham groups), risperidone (five in rTMS versus seven in sham groups), olanzapine (three versus zero), chlorpromazine (two versus one), ziprasidone (one versus zero), and sulpiride (one versus zero). Six patients were taking antidepressant medications including escitalopram oxalate, sertraline, and paroxetine. There were no any differences in the antipsychotics (type and dose) between two groups. In addition, chlorpromazine equivalent for every participant was calculated as described in other literatures in detail ${ }^{30}$.

\section{Clinical symptom and neuropsychological assessments}

The psychopathology of patients was assessed by three clinically trained staff using PANSS after a training session to ensure consistency and reliability of ratings. A PANSS fivefactor model of "positive", "negative", "cognitive", "emotional/ depressed", and "excited" was used in the current study ${ }^{31}$. The components are: positive component (P1 + P3 + P5 + G9); negative component (N1+ N2 + N3 + N4+ N6 + G7); cognitive component (P2 + N5 + G11); emotional/depressed 
component $(\mathrm{G} 2+\mathrm{G} 3+\mathrm{G} 6)$; and excited component (P4 + $\mathrm{P} 7+\mathrm{G} 8+\mathrm{G} 14)$.

The Repeatable Battery for the Assessment of Neuropsychological Status (RBANS) was used to assess the cognitive function, which contains five subtests of immediate and delayed memory, visuospatial/construction, attention, and language. A translated and adapted Chinese version of RBANS had been evaluated for reliability and clinical validity in our group ${ }^{32}$. Three psychologists assessed the cognitive impairment of the patients using the RBANS.

\section{Data analysis}

The data were described using the means \pm standard deviations and $p$ values $\leq 0.05$ were considered to be statistically significant. The demographic characteristics, clinical symptoms, and neuropsychological test scores were analyzed between two groups at baseline using analysis of variance (ANOVA) or the chi-square test. The intention-to-treat analysis was carried out and missing outcome data were entered as the principle of last observation carrying forward.

To investigate the effect of $20 \mathrm{~Hz}$ rTMS on cognitive performance and clinical symptoms in patients, the main strategy involved repeated-measures multivariate analyses of variance in the longitudinal study. The primary outcome was cognitive function measured by RBANS and clinical symptoms by PANSS. For the dependent variables, five time points (weeks $0,2,4,6$, and 8 ) were used as the repeated measures within-effect, and group $(20 \mathrm{~Hz}$ versus sham) was used as the between-effect. If the group $\times$ time interaction was significant, then the group differences at weeks 4,6 , and 8 were respectively analyzed by analysis of covariance (ANCOVA) and the baseline scores were covariates. If the above interaction effect was not significant, further statistical testing was not required.

The second aim was to determine whether improvement of cognitive function correlated with reduction of PANSS scores. Therefore, the changes in the RBANS scores and PANSS scores in the two groups (real/sham) before and after the 8-week treatment were calculated for each patient. Correlations between cognitive improvement and reductions in PANSS scores were analyzed and the Bonferroni corrections were applied when significant. Finally, we used multiple linear regressions to investigate the potential response predictors associated with changes in the cognitive scores and clinical symptoms.

\section{Results}

\section{Demographic and basic descriptive data}

At baseline, as shown in Tables 1 and 2, no difference was found between rTMS and sham groups in the demographic characters, clinical variables including the PANSS scores, as well as the RBANS total and subscores. Interestingly,
RBANS total score significantly negatively correlated with positive subscale score $(r=-0.284, \mathrm{df}=56, p=0.04)$, and negative subscale score $(r=-0.441, \mathrm{df}=56, p=0.001)$.

\section{rTMS treatment for cognitive performance}

During the treatment, 15 subjects were discontinued because they withdrew their consent (three in active and four in sham rTMS groups) and MRI images could not completely be restored to 3D stereo images (four in active and four in sham rTMS groups). Thus, 41 patients completed the clinical trial, including 21 in active and 20 in sham rTMS groups.

In the primary outcome, repeated-measures ANCOVA on the immediate memory revealed a significant time effect $(F=7.6, \mathrm{df}=2,74, p=0.009)$, a nonsignificant group effect $(F=2.1, \mathrm{df}=1,39, p=0.15)$, and a significant interaction effect $(F=6.3, \mathrm{df}=2,74, p=0.017)$. ANCOVA showed that the immediate memory performance was higher in the rTMS group compared with the sham group at week $8(F=6.1$, $\mathrm{df}=1,39, p=0.018$; $E S=0.36$ ), after covarying for education, age, and dose of drug (chlorpromazine equivalents).

Table 3 and Fig. 1 showed that the increase in immediate memory score from baseline to week 8 in the rTMS and sham groups was $28.2 \pm 19.3$ versus $16.0 \pm 10.9$, respectively $(F=6.3, \mathrm{df}=1,42, p=0.017)$. The mean difference in the change in the immediate memory index score from baseline to 8 weeks between the two groups was $12.2 \pm 8.4(\mathrm{ES}=0.78)$.

\section{rTMS treatment for psychopathological symptoms}

In the secondary outcome, changes in the PANSS and their subscale scores are illustrated in Table 2 and Fig. 2. Repeated-measures ANOVA on PANSS and all its subscales showed a marginally significant interaction effect (group $\times$ time: $F=2.2, \quad \mathrm{df}=1, \quad 39, \quad p=0.074$ ), nonsignificant group effect $(F=0.74, \mathrm{df}=1,39, p=0.48)$, and time effect $(F=1.6, \quad \mathrm{df}=4,39, \quad p=0.28)$. Further ANCOVA showed that the PANSS excited factor score was significantly more diminished in the rTMS group than in the sham group at week $6(F=3.5, \mathrm{df}=1,39, p=$ 0.07 ; $\mathrm{ES}=0.24)$ and at week $8(F=8.6, \mathrm{df}=1,39, p=$ $0.006 ; \mathrm{ES}=0.39$ ). The difference at week 8 remained significant after covarying for education, age, duration of illness, and dose of drug (chlorpromazine equivalents) $(F=7.4, \mathrm{df}=1,37, p=0.009)$.

No effect was found on the negative symptoms before and after treatment with rTMS after repeated-measures ANCOVA analysis.

\section{Correlation between the improvement of cognitive performance and change in psychopathological symptoms in $20 \mathrm{~Hz}$ rTMS group}

Correlation analysis in $20 \mathrm{~Hz}$ rTMS group showed significant correlations between the increase in immediate 
Table 1 Demographic data in active and sham groups.

\begin{tabular}{|c|c|c|c|c|c|c|}
\hline & $20 \mathrm{~Hz}(n=28)$ & Sham $(n=28)$ & $X^{2}$ or $F$ ( $p$ value $)$ & $20 \mathrm{~Hz}(n=21)$ & Sham $(n=20)$ & $X^{2}$ or $F$ ( $p$ value) \\
\hline Age (years) & $51.9 \pm 10.1$ & $56.0 \pm 7.3$ & $2.9(0.09)$ & $55.5 \pm 7.3$ & $49.3 \pm 10.2$ & $5.1(0.03)$ \\
\hline Education (years) & $7.9 \pm 2.6$ & $7.8 \pm 1.8$ & $0.01(0.99)$ & $7.9 \pm 1.8$ & $8.2 \pm 2.3$ & $0.1(0.71)$ \\
\hline Age of onset (years) & $20.1 \pm 2.4$ & $21.1 \pm 1.7$ & $0.2(0.86)$ & $21.1 \pm 1.8$ & $20.7 \pm 2.4$ & $0.4(0.55)$ \\
\hline Duration of illness & $31.3 \pm 9.7$ & $34.5 \pm 6.9$ & $2.4(0.95)$ & $34.3 \pm 6.8$ & $30.4 \pm 0.5$ & $2.1(0.16)$ \\
\hline Hospital time & $6.3 \pm 3.2$ & $5.6 \pm 3.1$ & $0.4(0.70)$ & $5.3 \pm 3.0$ & $5.5 \pm 2.6$ & $0.04(0.85)$ \\
\hline \multicolumn{7}{|l|}{ Antipsychotics } \\
\hline Clozapine & 16 & 20 & & 13 & 15 & \\
\hline Risperidone & 5 & 7 & & 3 & 4 & \\
\hline Olanzapine & 3 & 0 & & 3 & 0 & \\
\hline Chlorpromazine & 2 & 1 & & 2 & 1 & \\
\hline Sulpira & 1 & 0 & & 0 & 0 & \\
\hline Ziprasidone & 1 & 0 & & 0 & 0 & \\
\hline $\mathrm{DAD}(\mathrm{mg})$ & $413.9 \pm 226.5$ & $424.5 \pm 288.3$ & $1.2(0.31)$ & $420.4 \pm 221.3$ & $403.5 \pm 227.6$ & $1.6(0.23)$ \\
\hline PANSS total score & $72.3 \pm 12.9$ & $79.9 \pm 16.9$ & $2.3(0.10)$ & $75.1 \pm 14.9$ & $71.2 \pm 13.0$ & $0.8(0.38)$ \\
\hline P-subscore & $11.3 \pm 4.5$ & $11.4 \pm 3.6$ & $0.3(0.71)$ & $10.7 \pm 2.6$ & $11.5 \pm 4.8$ & $0.5(0.50)$ \\
\hline $\mathrm{N}$-subscore & $28.7 \pm 6.7$ & $31.9 \pm 8.4$ & $2.4(0.10)$ & $29.4 \pm 7.4$ & $27.4 \pm 6.3$ & $0.9(0.35)$ \\
\hline G-subscore & $32.3 \pm 7.6$ & $36.6 \pm 8.7$ & $2.4(0.10)$ & $34.9 \pm 8.3$ & $32.3 \pm 7.7$ & $1.2(0.29)$ \\
\hline RBANS total score & $58.3 \pm 12.1$ & $61.5 \pm 12.7$ & $0.8(0.37)$ & $60.1 \pm 12.5$ & $64.1 \pm 12.7$ & $1.1(0.31)$ \\
\hline Immediate memory & $52.0 \pm 12.9$ & $50.1 \pm 10.5$ & $0.2(0.83)$ & $51.8 \pm 10.9$ & $53.9 \pm 13.9$ & $0.3(0.59)$ \\
\hline Attention & $70.8 \pm 16.4$ & $66.8 \pm 13.5$ & $0.5(0.60)$ & $67.6 \pm 13.4$ & $74.6 \pm 14.8$ & $2.5(0.12)$ \\
\hline Visuospatial/ constructional & $72.2 \pm 17.2$ & $69.9 \pm 15.5$ & $0.2(0.85)$ & $72.9 \pm 14.8$ & $74.9 \pm 17.7$ & $0.2(0.70)$ \\
\hline Delayed memory & $65.4 \pm 20.1$ & $64.2 \pm 20.3$ & $0.1(0.96)$ & $66.8 \pm 20.1$ & $68.8 \pm 19.3$ & $0.1(0.75)$ \\
\hline Language & $79.3 \pm 15.2$ & $74.4 \pm 15.8$ & $0.7(0.48)$ & $75.8 \pm 15.6$ & $80.1 \pm 15.3$ & $0.8(0.38)$ \\
\hline
\end{tabular}

$D A D$ daily antipsychotic dose $(\mathrm{mg})$ (chlorpromazine equivalent), PANSS Positive and Negative Syndrome Scale, $P$ positive symptom, $N$ negative symptom, $G$ general psychopathology, RBANS repeatable battery for the assessment of neuropsychological status.

memory from baseline to week 8 and the following parameters: the reduction of PANSS excited factor score $(r=0.42, \mathrm{df}=21, p=0.05)$ and the positive subscore $(r=$ $0.56, \mathrm{df}=21, p=0.012$ ). However, none of these significances survived Bonferroni correction (all $p>0.05$ ).

Further analysis showed a trend toward significant association between increase in immediate memory index score and decrease of the positive subscore (beta $=7.5$, $t=2.03, p=0.07$ ), after covarying for education, age, and dose of treatment.

\section{Discussion}

The results of the current study showed that (1) rTMS produced an effective therapeutic benefit on immediate memory of patients with SCZ; (2) rTMS displayed an effect on PANSS excited factor, but not on negative symptoms; and (3) the improvement in immediate memory of patients was associated with the reduction in PANSS excited factor at 8 th week.
In this pilot double-blinded, randomized controlled trial, real rTMS significantly increased immediate memory score, demonstrating a clinically meaningful improvement of immediate memory performance in patients with SCZ in the $20 \mathrm{~Hz}$ rTMS group. Recently, rTMS has been proposed as a new treatment option for cognitive dysfunction, which alters the neuronal activity in the applied area and related areas ${ }^{6,18}$. Most of the literatures showed that rTMS was effective on attention and executive functions in certain patients with depression ${ }^{19}$. But in SCZ, only two studies have reported the promising effect of rTMS on cognitive function as compared with sham control. One earlier study, by Mogg et al., reported a positive effect of $10 \mathrm{~Hz}$ rTMS applied to left DLPFC on the California verbal learning test ${ }^{14}$. Interestingly, another recent study revealed that bilateral $20 \mathrm{~Hz}$ rTMS treatment of DLPFC for 4 weeks improved working memory. Particularly, rTMS significantly improved the accuracy of target response to a level comparable to healthy subjects 
Table 2 PANSS total score and subscores at baseline, week 2, week 4, week 6, and week 8 in 20 Hz rTMS and sham groups.

\begin{tabular}{|c|c|c|c|c|c|c|c|c|}
\hline & $\begin{array}{l}\text { Baseline } \\
(n=56)\end{array}$ & $\begin{array}{l}\text { Week } 2 \\
(n=41)\end{array}$ & $\begin{array}{l}\text { Week } 4 \\
(n=41)\end{array}$ & $\begin{array}{l}\text { Week } 6 \\
(n=41)\end{array}$ & $\begin{array}{l}\text { Week } 8 \\
(n=41)\end{array}$ & $\begin{array}{l}\text { Group } F \\
\text { ( } p \text { value) }\end{array}$ & $\begin{array}{l}\text { Time } F \\
\text { ( } p \text { value) }\end{array}$ & $\begin{array}{l}\text { Group } \times \text { time } F \\
(p \text { value })\end{array}$ \\
\hline \multicolumn{6}{|c|}{ PANSS total score } & $0.57(0.57)$ & $37.1(0.00)$ & $1.0(0.39)$ \\
\hline Sham & $79.9 \pm 16.9$ & $70.5 \pm 17.6$ & $67.8 \pm 20.3$ & $67.3 \pm 22.4$ & $66.8 \pm 22.7$ & & & \\
\hline $20 \mathrm{~Hz}$ & $72.3 \pm 12.9$ & $67.2 \pm 12.3$ & $62.8 \pm 13.7$ & $60.6 \pm 14.3$ & $59.2 \pm 14.4$ & & & \\
\hline \multicolumn{6}{|c|}{ P-subscore } & $0.54(0.58)$ & $0.04(0.83)$ & $0.06(0.94)$ \\
\hline Sham & $11.4 \pm 3.6$ & $10.4 \pm 2.6$ & $10.3 \pm 2.6$ & $10.1 \pm 2.8$ & $10.0 \pm 2.8$ & & & \\
\hline $20 \mathrm{~Hz}$ & $11.3 \pm 4.5$ & $11.4 \pm 4.8$ & $11.3 \pm 4.6$ & $10.9 \pm 4.3$ & $10.7 \pm 4.3$ & & & \\
\hline \multicolumn{6}{|c|}{ N-subscore } & $0.1(0.55)$ & $5.9(0.00)$ & $0.68(0.58)$ \\
\hline Sham & $31.9 \pm 8.4$ & $26.1 \pm 8.5$ & $24.8 \pm 9.3$ & $24.5 \pm 9.8$ & $23.8 \pm 10.0$ & & & \\
\hline $20 \mathrm{~Hz}$ & $28.7 \pm 6.7$ & $25.3 \pm 5.9$ & $22.7 \pm 6.2$ & $21.5 \pm 5.9$ & $20.9 \pm 5.9$ & & & \\
\hline \multicolumn{6}{|c|}{ G-subscore } & $1.3(0.28)$ & $16.8(0.00)$ & $1.3(0.28)$ \\
\hline Sham & $36.6 \pm 8.7$ & $34.0 \pm 8.6$ & $32.7 \pm 9.9$ & $32.7 \pm 10.8$ & $33.0 \pm 10.9$ & & & \\
\hline $20 \mathrm{~Hz}$ & $32.3 \pm 7.6$ & $30.6 \pm 7.1$ & $28.9 \pm 7.2$ & $28.3 \pm 7.3$ & $27.6 \pm 7.5$ & & & \\
\hline \multicolumn{6}{|c|}{ Postive factor } & $0.72(0.49)$ & $41.1(0.00)$ & $0.58(0.65)$ \\
\hline Sham & $5.9 \pm 2.5$ & $5.1 \pm 1.6$ & $5.0 \pm 1.4$ & $5.2 \pm 1.7$ & $5.2 \pm 1.7$ & & & \\
\hline $20 \mathrm{~Hz}$ & $6.6 \pm 3.4$ & $6.6 \pm 3.2$ & $6.7 \pm 3.1$ & $6.6 \pm 2.9$ & $6.6 \pm 3.0$ & & & \\
\hline \multicolumn{6}{|c|}{ Negative factor } & $0.72(0.49)$ & $41.1(0.00)$ & $0.58(0.65)$ \\
\hline Sham & $26.7 \pm 6.9$ & $21.9 \pm 7.5$ & $20.6 \pm 8.3$ & $20.0 \pm 8.5$ & $19.6 \pm 8.7$ & & & \\
\hline $20 \mathrm{~Hz}$ & $23.8 \pm 6.0$ & $20.8 \pm 5.7$ & $18.5 \pm 5.7$ & $17.5 \pm 5.4$ & $17.0 \pm 5.5$ & & & \\
\hline \multicolumn{6}{|c|}{ Cognitive factor } & $1.6(0.20)$ & $12.9(0.00)$ & $0.93(0.44)$ \\
\hline Sham & $11.1 \pm 3.8$ & $9.8 \pm 3.8$ & $9.3 \pm 4.2$ & $9.6 \pm 4.5$ & $9.3 \pm 4.8$ & & & \\
\hline $20 \mathrm{~Hz}$ & $8.8 \pm 2.5$ & $8.2 \pm 2.2$ & $7.7 \pm 2.5$ & $7.4 \pm 2.6$ & $7.3 \pm 2.7$ & & & \\
\hline \multicolumn{6}{|c|}{ Excited factor } & $0.74(0.48)$ & $1.6(0.28)$ & $2.2(0.074)$ \\
\hline Sham & $5.9 \pm 2.5$ & $5.5 \pm 1.7$ & $5.6 \pm 1.7$ & $5.6 \pm 1.7$ & $5.7 \pm 1.8$ & & & \\
\hline $20 \mathrm{~Hz}$ & $5.6 \pm 2.2$ & $5.3 \pm 1.2$ & $5.2 \pm 1.7$ & $4.8 \pm 1.3$ & $4.6 \pm 1.1$ & & & \\
\hline \multicolumn{6}{|c|}{ Depression factor } & $0.56(0.58)$ & $3.3(0.02)$ & $1.4(0.20)$ \\
\hline Sham & $4.4 \pm 1.9$ & $4.3 \pm 1.7$ & $4.3 \pm 1.7$ & $4.2 \pm 1.7$ & $4.2 \pm 1.6$ & & & \\
\hline $20 \mathrm{~Hz}$ & $4.1 \pm 1.9$ & $4.3 \pm 2.0$ & $3.9 \pm 1.9$ & $4.1 \pm 2.0$ & $3.9 \pm 2.0$ & & & \\
\hline
\end{tabular}

Table 3 Cognitive score and comparison at baseline and week 8 in $20 \mathrm{~Hz}$ and sham groups.

\begin{tabular}{|c|c|c|c|c|c|c|c|}
\hline & \multicolumn{2}{|c|}{ Baseline $(n=56)$} & \multicolumn{2}{|c|}{ Week $8(n=41)$} & \multirow{2}{*}{$\begin{array}{l}\text { Group } \\
F(p)\end{array}$} & \multirow{2}{*}{$\begin{array}{l}\text { Time } \\
F(p)\end{array}$} & \multirow{2}{*}{$\begin{array}{l}\text { Group } \times \text { time } \\
F(p \text { value })\end{array}$} \\
\hline & Sham & $20 \mathrm{~Hz}$ & Sham & $20 \mathrm{~Hz}$ & & & \\
\hline Immediate memory & $50.9 \pm 10.5$ & $52.0 \pm 12.9$ & $69.7 \pm 21.4$ & $82.1 \pm 21.6$ & $3.3(0.077)$ & $52.1(<0.001)$ & $6.3(0.01)$ \\
\hline Attention & $63.1 \pm 15.4$ & $70.5 \pm 13.3$ & $66.8 \pm 13.5$ & $70.8 \pm 16.4$ & $3.4(0.07)$ & $4.5(0.04)$ & $0.03(0.96)$ \\
\hline Visuospatial/constructional & $69.9 \pm 15.5$ & $72.2 \pm 17.2$ & $78.5 \pm 13.3$ & $82.1 \pm 18.6$ & 0.430 .52 & 10.10 .003 & $0.08(0.8)$ \\
\hline Delayed memory & $64.2 \pm 20.3$ & $65.4 \pm 20.1$ & $86.0 \pm 18.5$ & $90.7 \pm 21.1$ & $0.35(0.56)$ & $70.4(<0.001)$ & $0.34(0.57)$ \\
\hline Language & $74.4 \pm 15.8$ & $79.3 \pm 15.2$ & $85.8 \pm 17.0$ & $84.6 \pm 9.5$ & $0.28(0.60)$ & $7.0(0.012)$ & $1.59(0.22)$ \\
\hline RBANS total score & $58.3 \pm 12.1$ & $61.5 \pm 12.7$ & $71.3 \pm 14.8$ & $78.1 \pm 16.2$ & $2.5(0.13)$ & $81.0(<0.001)$ & $2.6(0.11)$ \\
\hline
\end{tabular}




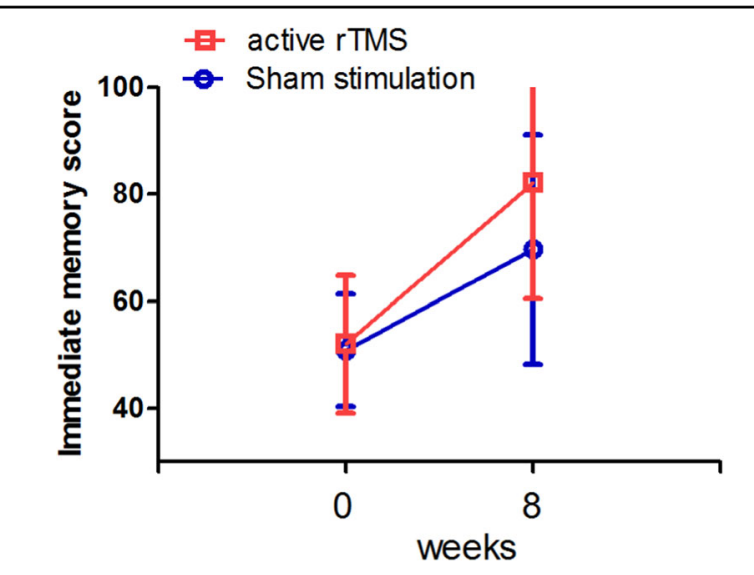

Fig. 1 Comparison of immediate memory score between rTMS and sham groups before and after 8 weeks of treatment. rTMS treatment significantly increased the immediate memory score in the patients with schizophrenia, as compared with sham stimulation $(p<$ 0.05).

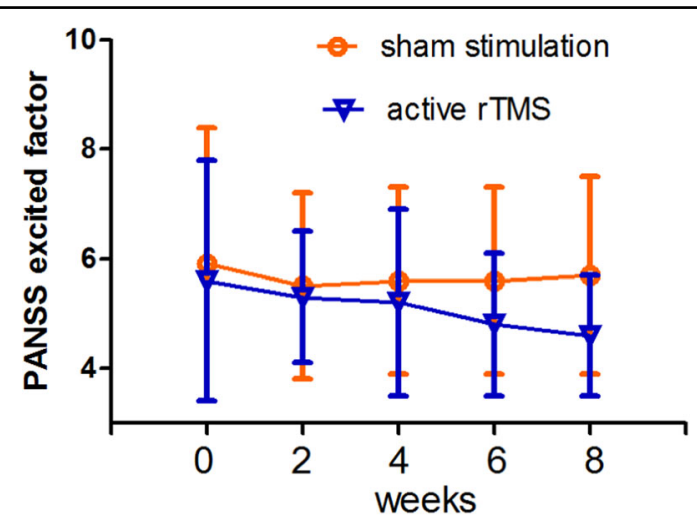

Fig. 2 Comparison of PANSS excited factor between rTMS and sham groups before and after 8 weeks of treatment. rTMS treatment displayed a trendency to significance for PANSS excited factor in the patients with schizophrenia, compared with sham stimulation $(p=0.074)$.

in the 3-back task in patients ${ }^{15}$. Our findings are similar to those reported in the above study in several points: first, the patients recruited in our study also had predominant negative symptoms; second, our results indicated that $20 \mathrm{~Hz}$ rTMS targeting on left DLPFC improved immediate memory performance in patients.

The exact mechanisms responsible for the effective treatment of rTMS for cognitive impairment are still unknown. Preclinical studies have shown that rTMS causes complex biochemical effects and neurotropic effects by reducing inflammation and oxidative stress ${ }^{33,34}$. For example, studies have revealed that rTMS increases the release of dopamine in certain brain pathways ${ }^{35}$, which is consistent with the hypoactive dopaminergic hypothesis in cognitive deficits of patients with $\mathrm{SCZ}^{36}$.
Moreover, animal studies found that rTMS altered NMDA receptor concentration after a single stimulation, functionally as a potential agonist of the receptors ${ }^{37}$. Thus, the agonistic effect and upregulation of NMDA receptors by rTMS may improve the cognitive impairment. Second, a recent study showed that high-frequency rTMS may improve the impaired neuronal plasticity by activating BDNF pathway in ischemic rats ${ }^{38}$. Thus, highfrequency rTMS may improve cognitive deficits in SCZ patients by activation of neurotrophic factors, which play critical roles in neuroplasticity in the hippocampus related to learning and memory ${ }^{39}$. In sum, the potential mechanism of rTMS treatment on cognitive dysfunction may be due to those molecular effects to decorate the properties of neurons and increase the expression of neurotransmitters and their receptors, as well as activate neurotrophic factors ${ }^{33}$. However, these points are only our speculations since we did not measure the neurobiochemicals in the patients. The exact mechanisms underlying the effective treatment of $20 \mathrm{~Hz}$ rTMS for cognitive deficits in SCZ need further investigation.

Another finding in the present study was that $20 \mathrm{~Hz}$ rTMS over left DLPFC for 8 consecutive weeks displayed a trendency to significantly better therapeutic effect on excited factor of PANSS. Failure to improve the negative symptoms after treatment with rTMS is consistent with recent studies in other groups using rTMS targeting to left DLPFC for negative symptoms in patients with $\mathrm{SCZ}^{12}$. However, four other meta-analyses found significant effects of rTMS for negative symptoms of $\mathrm{SCZ}^{7,8,40,41}$. The discrepancies in the therapeutic effects of rTMS for clinical symptoms of the patients may be caused by duration of illness, different stages of disease progression (chronic versus acute), adjunctive antipsychotic medication, the type of outcome measures used, duration of rTMS treatment, as well as the techniques of rTMS, such as stimulus frequency, position and intensity of treatment, as well as the shape and dimension of coils.

Interestingly, we established a superior effect of active rTMS on the PANSS excited factor. Our further ANOVA analysis showed a significant difference in time $\times$ group interaction on poor impulse control $(p<0.05)$, indicating that the $20 \mathrm{~Hz}$ rTMS treatment has a positive effect on the PANSS excited factors compared with sham stimulation. A recent exploratory secondary analysis of the data from "rTMS for the Treatment of Negative Symptoms in Schizophrenia" (RESIS) trial reported that real rTMS significantly improved the PANSS excited factor, which was in accordance with our results ${ }^{42}$. However, further analysis in our study and the previous reanalysis study did not pass the Bonferroni corrections. This was due to the excited factor score consisting of four items of clinical symptoms that were already quite low prior to the treatment. Further longitudinal studies with larger samples in 
SCZ are warranted. Specifically, we found that the increase in the immediate memory score was correlated with the reduction in PANSS excited score at week 8, suggesting a close relationship between the improvements of cognitive deficits and clinical symptoms in patients with SCZ. At baseline, we found significant associations between the RBANS score and PANSS negative and positive scores, which provided further evidence for this point. Many studies support the notion that cognitive dysfunction is correlated with certain clinical symptoms in SCZ, indicating that they share common pathological mechanism $^{43}$.

It is known that dopaminergic dysfunctions are associated with cognitive deficits and poor impulse control of $\mathrm{SCZ}^{44}$. Impaired ventral prefrontal cortex and DLPFC are interconnected with impulse controls ${ }^{45}$. Recent studies also showed that prefrontal dopaminergic dysfunction could lead to disturbances in striatal dopamine. Conversely, the striatal dopaminergic disturbances could cause the aggressive behaviors. Recent studies also supported the proposal that $\mathrm{D}_{3}$-preferred $\mathrm{D}_{2} / \mathrm{D}_{3}$ agonists are associated with impulsive control. As mentioned above, high-frequency rTMS could cause the release of dopamine in the mesostriatal brain pathways ${ }^{46}$, which may improve poor impulse control and cognitive deficits simultaneously. Indeed, we found a close relationship between the improvements of excited factor and cognitive deficits of SCZ with rTMS treatment in our present study. However, the mechanisms underlying how the rTMS treatment may influence DA system and further improve clinical symptoms and cognitive deficits of patients with SCZ warrant further investigation.

The limitations of our current study were shown as follows. First, we had a relatively small sample size and may have led to false negative or positive results. Our findings should be confirmed in a large sample from different ethnic populations. Second, one important limitation of our study was the limited 8-week treatment period and no follow-up, which may not be long enough to assess changes in multiple domains of cognition. Therefore, a follow-up study to investigate the efficacy of rTMS for clinical symptoms and cognitive impairments of SCZ is needed to explore their relationship. Third, the subjects in our study were chronically hospitalized older patients, with a longer illness duration and more severe psychopathology than first episode and drug-naive patients with SCZ or typical psychotic outpatients. This limits the generalization of our findings in this study. Fourth, before this study, we only asked the patients verbally whether they had major life stressor or clinical significant emotional disturbance; however, we did not assess these questions by using a rating scale. Fifth, considering that bilateral stimuli reported in the literatures were more likely to improve cognitive functions such as working memory in SCZ, only the left DLPFC was stimulated in the present study, which was a limitation. Sixth, drug use may have an impact on the cognitive function of patients. In this study, however, substance exposure was not assessed by urine analysis or other methods, but only by self-reported drug use.

In summary, the present study indicates that $20 \mathrm{~Hz}$ rTMS treatment is beneficial for clinical symptoms and cognitive dysfunction of SCZ. The potential efficacy of $20 \mathrm{~Hz}$ rTMS for cognitive dysfunction is important for clinical utilization, since cognitive impairments have been shown to be one of the major obstacles to social dysfunction rehabilitation in patients with SCZ. Thus, rTMS may be a promising cognitive enhancing tool for patients with SCZ as well as other mental disorders. Also, we have found that $20 \mathrm{~Hz}$ rTMS is an effective treatment for excited symptoms of SCZ, especially impulse control. Although our findings are encouraging, further investigations are necessary to confirm its efficacy for cognitive deficits in a large sample size of first episode and drug-naïve schizophrenia patients in different ethnic populations with a long follow-up period using a longitudinal design.

\section{Acknowledgements \\ H.Y.G., S.P.T., Y.P.R., M.H.X. and X.Y.Z. were responsible for study design, statistical analysis, and manuscript preparation. J.M.Z., K.Q.W., X.R.S., Y.F.P., J.M.G., L.J., Y.H.W., H.Y.L., S.G.S., H.R.W., H.S.G., X.W.L., H.J.Y. and B.C.W. were responsible for recruiting the patients, performing the clinical rating, and collecting the clinical data. X.P.L., H.E.W., M.H.X. and X.Y.Z. were involved in evolving the ideas and editing the manuscript. H.E.W. and M.H.X. were involved in writing the protocol and cowrote the paper. All authors have contributed to and have approved the final manuscript.}

\section{Funding}

This study was supported by the Scientific and Technological Program of Hebei (20150593). These sources had no further role in study design; in the collection, analysis and interpretation of data; in the writing of the report; and in the decision to submit the paper for publication.

\section{Author details}

${ }^{1}$ Hebei Province Rong-Jun Hospital, Baoding, China. ${ }^{2}$ The National Clinical Research Center for Mental Disorders \& Beijing Key Laboratory of Mental Disorders, Beijing Anding Hospital, Capital Medical University, Beijing, China. ${ }^{3}$ Department of Psychiatry and Behavioral Sciences, Baylor College of Medicine, Houston, TX, USA. ${ }^{4}$ Peking University Huilongguan Clinical Medical School, Beijing Huilongguan Hospital, Beijing, China. ${ }^{5}$ Department of Psychology, University of Chinese Academy of Sciences, Beijing, China. ${ }^{6}$ CAS Key Laboratory of Mental Health, Institute of Psychology, Chinese Academy of Sciences, Beijing, China

\section{Conflict of interest}

The authors declare that they have no conflict of interest.

\section{Publisher's note}

Springer Nature remains neutral with regard to jurisdictional claims in published maps and institutional affiliations.

Received: 24 July 2019 Revised: 5 November 2019 Accepted: 8 November 2019

Published online: 25 February 2020 


\section{References}

1. Misiak, B., Bienkowski, P. \& Samochowiec, J. Cariprazine-a novel antipsychotic drug and its place in the treatment of schizophrenia. Psychiatr. Pol. 52 971-981 (2018)

2. Van Rheenen, T. E. et al. Characterizing cognitive heterogeneity on the schizophrenia-bipolar disorder spectrum. Psychol. Med. 47, 1848-1864 (2017)

3. Fatouros-Bergman, H., Cervenka, S., Flyckt, L., Edman, G. \& Farde, L. Metaanalysis of cognitive performance in drug-naive patients with schizophrenia. Schizophr. Res. 158, 156-162 (2014).

4. Fioravanti, M., Bianchi, V. \& Cinti, M. E. Cognitive deficits in schizophrenia: an updated metanalysis of the scientific evidence. BMC Psychiatry 12, 64 (2012).

5. Mclntyre, R. S. et al. Cognitive deficits and functional outcomes in major depressive disorder: determinants, substrates, and treatment interventions. Depress. Anxiety 30, 515-527 (2013).

6. Francis, M. M., et al. Cognitive effects of bilateral high frequency repetitive transcranial magnetic stimulation in early phase psychosis: a pilot study. Brain Imaging Behav. 13, 852-861 (2019).

7. Slotema, C. W., Blom, J. D., Hoek, H. W. \& Sommer, I. E. Should we expand the toolbox of psychiatric treatment methods to include repetitive transcranial magnetic stimulation (rTMS)? A meta-analysis of the efficacy of rTMS in psychiatric disorders. J. Clin. Psychiatry 71, 873-884 (2010).

8. Shi, C., Yu, X., Cheung, E. F., Shum, D. H. \& Chan, R. C. Revisiting the therapeutic effect of rTMS on negative symptoms in schizophrenia: a meta-analysis. Psychiatry Res. 215, 505-513 (2014).

9. Slotema, C. W., Aleman, A., Daskalakis, Z. J. \& Sommer, I. E. Meta-analysis of repetitive transcranial magnetic stimulation in the treatment of auditory verbal hallucinations: update and effects after one month. Schizophr. Res. 142, 40-45 (2012).

10. Hovington, C. L., McGirr, A., Lepage, M. \& Berlim, M. T. Repetitive transcranial magnetic stimulation (rTMS) for treating major depression and schizophrenia: a systematic review of recent meta-analyses. Ann. Med. 45, 308-321 (2013).

11. Aleman, A., Enriquez-Geppert, S., Knegtering, H. \& Dlabac-de Lange, J. J. Moderate effects of noninvasive brain stimulation of the frontal cortex for improving negative symptoms in schizophrenia: meta-analysis of controlled trials. Neurosci. Biobehav. Rev. 89, 111-118 (2018).

12. $\mathrm{He}, \mathrm{H}$. et al. Repetitive transcranial magnetic stimulation for treating the symptoms of schizophrenia: a PRISMA compliant meta-analysis. Clin. Neurophysiol. 128, 716-724 (2017).

13. Hoffman, R. E. et al. Temporoparietal transcranial magnetic stimulation for auditory hallucinations: safety, efficacy and moderators in a fifty patient sample. Biol. Psychiatry 58, 97-104 (2005).

14. Mogg, A. et al. Repetitive transcranial magnetic stimulation for negative symptoms of schizophrenia: a randomized controlled pilot study. Schizophr. Res. 93, 221-228 (2007).

15. Barr, M. S. et al. Can repetitive magnetic stimulation improve cognition in schizophrenia? Pilot data from a randomized controlled trial. Biol. Psychiatry $\mathbf{7 3}$ 510-517 (2013)

16. Oh, S. Y. \& Kim, Y. K. Adjunctive treatment of bimodal repetitive transcranial magnetic stimulation (rTMS) in pharmacologically non-responsive patients with schizophrenia: a preliminary study. Prog. Neuropsychopharmacol. Biol. Psychiatry 35, 1938-1943 (2011).

17. Wolwer, W. et al. Repetitive transcranial magnetic stimulation (rTMS) improves facial affect recognition in schizophrenia. Brain Stimul. 7, 559-563 (2014).

18. Hasan, A. et al. Cognitive effects of high-frequency rTMS in schizophrenia patients with predominant negative symptoms: results from a multicenter randomized sham-controlled trial. Schizophr. Bull. 42, 608-618 (2016).

19. limori, T. et al. Effectiveness of the prefrontal repetitive transcranial magnetic stimulation on cognitive profiles in depression, schizophrenia, and Alzheimer's disease: a systematic review. Prog. Neuropsychopharmacol. Biol. Psychiatry $\mathbf{8 8}$ 31-40 (2019).

20. Coles, A. S., Kozak, K. \& George, T. P. A review of brain stimulation methods to treat substance use disorders. Am. J. Addict. 27, 71-91 (2018).

21. Dollfus, S. et al. High-frequency neuronavigated rTMS in auditory verbal hallucinations: a Pilot Double-Blind Controlled Study in patients with schizophrenia. Schizophr. Bull. 44, 505-514 (2018).

22. Lara, A. H. \& Wallis, J. D. The role of prefrontal cortex in working memory: a mini review. Front Syst. Neurosci. 9, 173 (2015).

23. Janicak, P. G. et al. Durability of clinical benefit with transcranial magnetic stimulation (TMS) in the treatment of pharmacoresistant major depression: assessment of relapse during a 6-month, multisite, open-label study. Brain Stimul. 3, 187-199 (2010).

24. Leyman, L., De Raedt, R., Vanderhasselt, M. A. \& Baeken, C. Effects of repetitive transcranial magnetic stimulation of the dorsolateral prefrontal cortex on the attentional processing of emotional information in major depression: a pilot study. Psychiatry Res. 185, 102-107 (2011).

25. O'Reardon, J. P. et al. Efficacy and safety of transcranial magnetic stimulation in the acute treatment of major depression: a multisite randomized controlled trial. Biol. Psychiatry 62, 1208-1216 (2007).

26. Peleman, K. et al. Using 3D-MRI to localize the dorsolateral prefrontal cortex in TMS research. World J. Biol. Psychiatry 11, 425-430 (2010).

27. Ahdab, R., Ayache, S. S., Brugieres, P., Goujon, C. \& Lefaucheur, J. P. Comparison of "standard" and "navigated" procedures of TMS coil positioning over motor, premotor and prefrontal targets in patients with chronic pain and depression. Neurophysiol. Clin. 40, 27-36 (2010).

28. Pommier, B. et al. Easy methods to make the neuronavigated targeting of DLPFC accurate and routinely accessible for rTMS. Neurophysiol. Clin. 47, 35-46 (2017).

29. Smith, J. E. \& Peterchev, A. V. Electric field measurement of two commercial active/sham coils for transcranial magnetic stimulation. J. Neural Eng. 15, 054001 (2018).

30. Leucht, S., Samara, M., Heres, S. \& Davis, J. M. Dose equivalents for antipsychotic drugs: the DDD method. Schizophr. Bull. 42(Suppl 1), S90-594 (2016).

31. Wallwork, R. S., Fortgang, R., Hashimoto, R., Weinberger, D. R. \& Dickinson, D. Searching for a consensus five-factor model of the Positive and Negative Syndrome Scale for schizophrenia. Schizophr. Res. 137, 246-250 (2012).

32. Zhang, B. H., Tan, Y. \& Zhang, W. Repeatable battery for the assessment of neuropsychologicla status (RBANS) as a screening test in Chinese: reliability and vaidity. J. Chin. Ment. Health 28, 865-869 (2009).

33. Cirillo, G. et al. Neurobiological after-effects of non-invasive brain stimulation. Brain Stimul. 10, 1-18 (2017).

34. Soundara Rajan, T. et al. Mechanism of action for rTMS: a working hypothesis based on animal studies. Front. Physiol. 8, 457 (2017).

35. Lanza, G. et al. Clinical and electrophysiological impact of repetitive lowfrequency transcranial magnetic stimulation on the sensory-motor network in patients with restless legs syndrome. Ther. Adv. Neurol. Disord. 11 1756286418759973 (2018).

36. Topolov, M. K. \& Getova, D. P. Cognitive impairment in schizophrenia, neurotransmitters and the new atypical antipsychotic aripiprazole. Folia Med. 58, 12-18 (2016).

37. Ragert, P. et al. Combination of $5 \mathrm{~Hz}$ repetitive transcranial magnetic stimulation (rTMS) and tactile coactivation boosts tactile discrimination in humans. Neurosci. Lett. 348, 105-108 (2003).

38. Luo, J., et al. High-frequency repetitive transcranial magnetic stimulation (rTMS) improves functional recovery by enhancing neurogenesis and activating BDNF/TrkB signaling in ischemic rats. Int J Mol Sci. 18, E455 (2017).

39. Leal, G., Afonso, P. M., Salazar, I. L. \& Duarte, C. B. Regulation of hippocampal synaptic plasticity by BDNF. Brain Res. 1621, 82-101 (2015).

40. Freitas, C., Fregni, F. \& Pascual-Leone, A. Meta-analysis of the effects of repetitive transcranial magnetic stimulation (rTMS) on negative and positive symptoms in schizophrenia. Schizophr. Res. 108, 11-24 (2009).

41. Dlabac-de Lange, J. J., Knegtering, R. \& Aleman, A. Repetitive transcranial magnetic stimulation for negative symptoms of schizophrenia: review and meta-analysis. J. Clin. Psychiatry 71, 411-418 (2010).

42. Hansbauer, M. et al. Efficacy of high-frequency repetitive transcranial magnetic stimulation on PANSS factors in schizophrenia with predominant negative symptoms - results from an exploratory re-analysis. Psychiatry Res. 263, 22-29 (2018).

43. Maia, T. V. \& Frank, M. J. An integrative perspective on the role of dopamine in schizophrenia. Biol. Psychiatry 81, 52-66 (2017).

44. Corvol, J. C. et al. Longitudinal analysis of impulse control disorders in Parkinson disease. Neurology 91, e189-e201 (2018).

45. Aron, A. R., Robbins, T. W. \& Poldrack, R. A. Inhibition and the right inferior frontal cortex. Trends Cogn. Sci. 8, 170-177 (2004).

46. Strafella, A. P., Paus, T., Barrett, J. \& Dagher, A. Repetitive transcranial magnetic stimulation of the human prefrontal cortex induces dopamine release in the caudate nucleus. J. Neurosci. 21, RC157 (2001). 